\title{
Investigation on Over-charging Characteristics of Lithium Iron Phosphate Battery
}

\author{
You-Chun Huang1, Pei-Lin Chen², Yao-Ching Hsieh', Hung-Liang Cheng ${ }^{3}$, Chin-Sien Moo ${ }^{1}$ \\ ${ }^{1}$ Department of Electrical Engineering/National Sun Yat-sen University \\ 70 Lienhai Rd., Kaohsiung 80424, Taiwan \\ d073010002@student.nsysu.edu.tw; ychsieh@mail.ee.nsysu.edu.tw; mooxx@mail.ee.nsysu.edu.tw \\ ${ }^{2}$ Certification Department/Mean Well Enterprises Co. \\ No.28, Wuquan 3rd Rd., Wugu Dist., New Taipei City 24891, Taiwan \\ alison@meanwell.com \\ ${ }^{3}$ Department of Electrical Engineering/I-Shou University \\ No.1, Sec. 1, Syuecheng Rd., Dashu District, Kaohsiung City 84001,Taiwan \\ hlcheng@isu.edu.tw
}

\begin{abstract}
To characterize the safe operation region of the lithium iron phosphate battery, experiments are conducted for over-charging to realize the borderline of the fatal damage as well as the detrimental effects to the state-of-health ( $\mathrm{SOH}$ ). An excessive amount of charge can be carefully brought into the battery with an extensive upper limit of the charging voltage under the limitation of an acceptable charge volume. The test results evidences that the utilizable capacity of a lithium iron phosphate battery can be approximately extended by $8 \%$ as compared with the conventionally recommended standard constant-current constant-voltage (CC-CV) charging regime.
\end{abstract}

Keywords: Lithium iron phosphate (LiFePo4) battery, Over charging, State of health ( $\mathrm{SOH})$, Constant-current constantvoltage $(\mathrm{CC}-\mathrm{CV})$ charging.

\section{Introduction}

With great improvements in material technology and advancements in manufacturing processes, rechargeable batteries are now with more satisfactory performances and reasonable costs. They are now playing the most important role in our modern daily life, not only for powering the portable electronic devices, such as laptops, smart phones, digital cameras and so on, but also for electric vehicles which have become more and more popular. In addition, they are the essential parts in the distributed green power systems for energy storage [1-3].

As compared to many other kinds of rechargeable batteries, lithium-ion batteries have the advantages of high energy and power densities, no memory effect and low self-discharge. In practice, a variety of lithium-ion batteries with distinctive chemistries have been employed for different purposes with required performances and acceptable costs. Among which, the lithium iron phosphate (LiFePO4) battery uses a lithium-ion-derived chemistry and shares many advantages. As compared with other lithium-ion battery chemistries, it has a relatively higher power density. In other words, it has a higher current or peak-power ratings. Moreover, it provides a nearly constant voltage over the dischargeable region and offers a much longer cycle life with less risk of fire or explosion. These merits make it a good potential in many applications especially for the replacement of lead-acid batteries [4-6].

In general, the loaded voltage of a lithium iron phosphate cell is normalized as $3.2 \mathrm{~V}$ or $3.3 \mathrm{~V}$ during discharging. The discharged battery cell is regarded being exhausted when the terminal voltage has reached the low limit. On the other hand, an upper limit is set during the charging phase for the standard constant-current constant-voltage (CC-CV) charging regime. A battery is noted to have been over discharged in the case that the loaded voltage drops below the lower limit. On the other hand, it is noted being over charged when the terminal voltage becomes higher than the upper limit during the charging phase $[7,8]$.

With a standard CC-CV charging regime, it takes a noticeably long time with a lower energy efficiency to fully charge the battery at the CV stage. To shorten the charging time and at the same time to improve the energy efficiency, one can 
make use of only the $\mathrm{CC}$ stage during the charging period. However, this may sacrifice a part of the available capacity by excluding the $\mathrm{CV}$ charging stage.

\section{Definitions and Specifications for Tested Battery}

The definitions for related parameters and nomenclatures for testing lithium iron phosphate batteries are explained as the following.

(1) Nominal capacity, $Q_{N}$ : The releasable electrical energy of a new battery claimed by the manufacturer.

(2) Original capacity, $Q_{O}$ : The practical accessible charge of a new battery obtained by averaging the capacities tested from several standard charging-discharging cycles.

(3) Existing capacity, $Q_{C}$ : The currently remaining capacity of an aged battery.

(4) Extended capacity, $Q_{H}$ : The accessible charge of a battery which has been overly charged.

(5) State-of-charge (SOC): The percentage of the remaining releasable charge of a battery relative to the existing capacity.

(6) State-of-health ( $\mathrm{SOH}$ ): The percentage of the existing capacity relative to the original capacity.

(7) Holding voltage, $V_{H}$ : The upper voltage limit at the CV stage.

(8) Cut-off current, $I_{H}$ : The designated current at the end of the CV stage to stop the charging phase.

(9) Cut-off voltage, $V_{C}$ : The lower limit of the loaded voltage, at which a battery is regarded as completely discharged.

(10) Charging-discharging efficiency, $\eta_{e}$ : The ratio between the released charge during the discharging phase and the received charge during the last charging phase in a full charging-discharging cycle.

(11) Overcharged capacity, $Q_{A}$ : The total electrical energy needed for overcharging.

(12) Overcharged capacity, $Q_{R}$ : The releasable capacity from the overcharged battery.

Table I lists the specifications of three brands of tested batteries, which are with single lithium iron phosphate cells. In the paper, they are named by A, S and K, respectively. The nominal capacities given by the manufacturers are $2.5 \mathrm{Ah}, 2.85$ $\mathrm{Ah}$ and 2.6 Ah, respectively. In practical applications, however, their releasable charges may be very different with the claimed capacities. Instead of the nominal capacity given by the manufacturer, the original capacity of a new battery and the existing capacity of an aged battery are obtained by the standard charging and discharging profiles as illustrated by Figure 1.

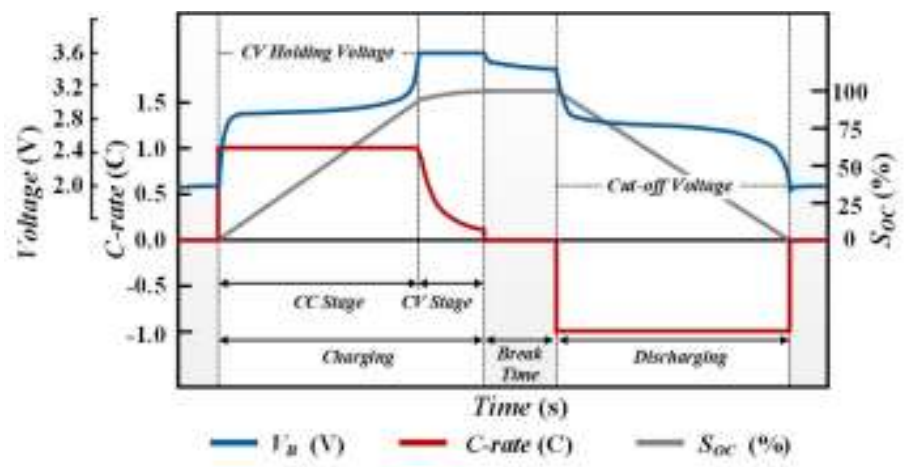

Fig. 1: Standard charging/discharging regime for lithium iron phosphate battery.

Table 1: Specifications of the tested batteries.

\begin{tabular}{|c|c|c|c|}
\hline Tested Battery & A & S & K \\
\hline Nominal Capacity & $2.5 \mathrm{Ah}$ & $2.85 \mathrm{Ah}$ & $2.6 \mathrm{Ah}$ \\
\hline Original Capacity & $2611 \mathrm{mAh}$ & $2805 \mathrm{mAh}$ & $2211 \mathrm{mAh}$ \\
\hline Nominal Voltage & $3.3 \mathrm{~V}$ & $3.2 \mathrm{~V}$ & $3.2 \mathrm{~V}$ \\
\hline Holding Voltage & $3.6 \mathrm{~V}$ & $3.6 \mathrm{~V}$ & $3.65 \mathrm{~V}$ \\
\hline Cut-off Voltage & $2 \mathrm{~V}$ & $2 \mathrm{~V}$ & $2.5 \mathrm{~V}$ \\
\hline $\begin{array}{c}\text { Charging-discharging } \\
\text { Efficiency }\end{array}$ & $99.86 \%$ & $99.94 \%$ & $99.99 \%$ \\
\hline
\end{tabular}


The discharging current and the constant current at the $\mathrm{CC}$ stage for charging are both set at $1 \mathrm{C}$. A cut-off current of $0.1 \mathrm{C}$ at the $\mathrm{CV}$ stage of the standard CC-CV charging regime is recommended for the lithium iron phosphate cell. The upper limit of the holding voltage is recommended at $3.6 \mathrm{~V}$ for Batteries A and $\mathrm{S}$, and $3.65 \mathrm{~V}$ for Battery $\mathrm{K}$. The cut-off voltage for the constant current discharging is limited by $2 \mathrm{~V}$ for Batteries $\mathrm{A}$ and $\mathrm{S}$, and $2.5 \mathrm{~V}$ for Battery $\mathrm{K}$.

Figure 2 shows the three curves of charging voltages for the three tested batteries. The battery capacity is the maximum accessible charge acquired by cumulating the charges from either an empty-to-full charging phase or a full-to-empty discharging phase. For a lithium iron phosphate battery with a high charging-discharging columbic efficiency, the trivial difference between the chargeable and releasable capacities on subsequent phases can be ignored. As indicated in Table I, the original capacities obtained from the standard charging-discharging cycles are $2611 \mathrm{~mA}, 2805 \mathrm{~mA}$ and $2211 \mathrm{~mA}$, respectively.

It should be noted that the battery capacity decays as the operating time elapses. As a result, the charging/discharging current may be miscalculated by the C-rate with the battery's nominal capacity or original capacity. To evaluate the battery performances in a more accurate manner, the C-rate has to be rescaled time to time in accordance with the latest existing capacity.

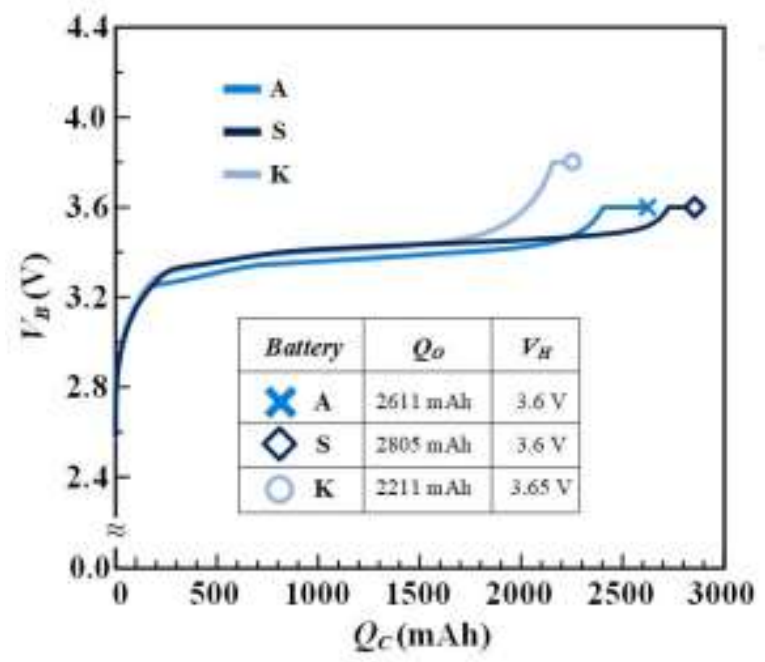

Fig. 2: Charging characteristic of the tested batteries.

\section{Over-charging Experiments}

In the case that a higher holding voltage is applied to the battery at the CV stage in the CC-CV charging method, an excessive amount of charges is brought into the battery. Over-charging on a battery may cause an abnormal operation. In the over-charging experiment, the tested cells are charged by the CC-CV method with a constant current of $1 \mathrm{C}$ and a holding voltage set at a relatively high level of $4.75 \mathrm{~V}$. With such a high holding voltage, the abnormal charging may happen either at the CC stage or the CV stage.

In the test case of Figure 3, the battery voltage increases first rapidly and then goes to the flat-voltage region since it is charged up from the empty status by a constant current of $1 \mathrm{C}$. The voltage increases fast again when the cell has received an amount of charge just about $95 \%$ of the rated capacity. The cell voltage does not continue to rise after reaching a peak voltage of $4.7 \mathrm{~V}$. At this peak point, an amount of $2926 \mathrm{mAh}$ has been charged to the tested cell. Beyond the peak, the cell voltage starts to decrease. Definitely, the tested cell will be damaged if a constant current is continuously applied to it.

Figure 4 shows another abnormal case of over-charging. In this test, the cell voltage increases up to the holding voltage of $4.75 \mathrm{~V}$ after going through the flat-voltage region. The tested cell stays at this voltage and the charging current decreases just like that for the standard CC-CV regime. The abnormal phenomenon happens when the cell is charged to $3032 \mathrm{mAh}$. At this point, the charging current starts to increase drastically. Certainly, the sharply rising current will cause a damage of the tested cell. 
Damages caused by over-charging to the lithium iron phosphate batteries can be classified into three degrees in accordance with the changes of surface appearances on the battery packs as shown in Figure 5. A swelling end on the battery pack is found since it is lightly damaged. The batteries with such a damage can be normally operated for a long life cycle. A more severe over-charging will cause an opening of the pressure relief valve. These batteries can be charged and discharged for a number of cycles but with fast fading in the capacity. Scorched surfaces on the battery packs with electrolyte leakage can be found for the harshly damaged batteries.

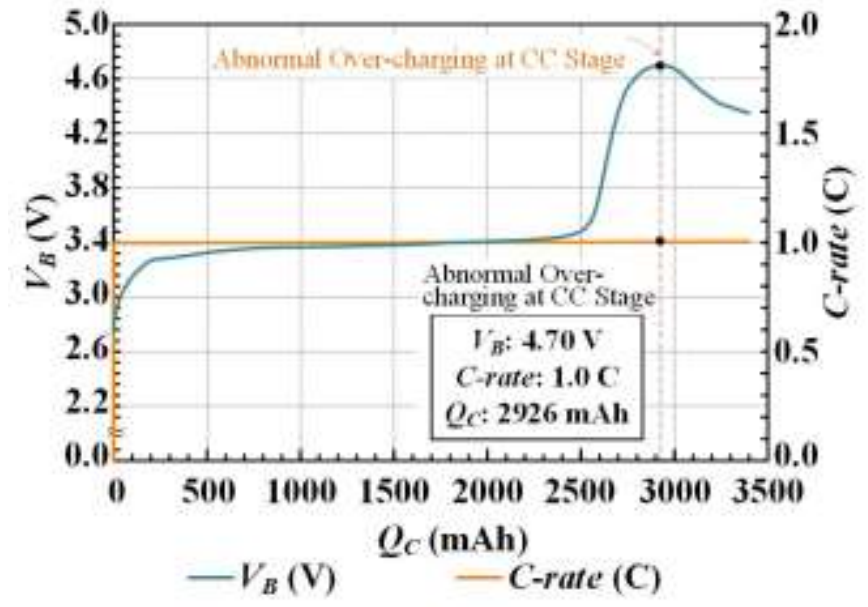

Fig. 3: Abnormal over-charging at the CC stage.

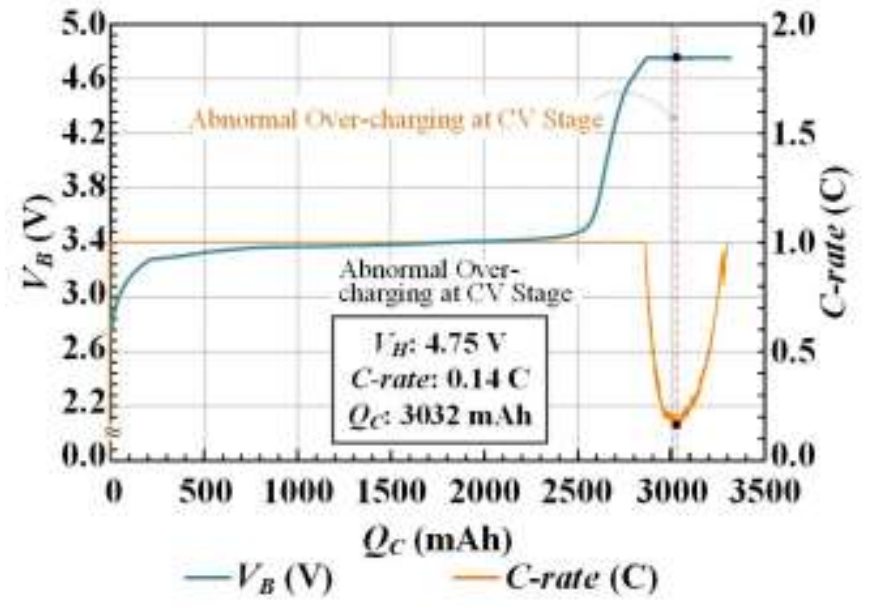

Fig. 4: Abnormal over-charging at the CV stage.

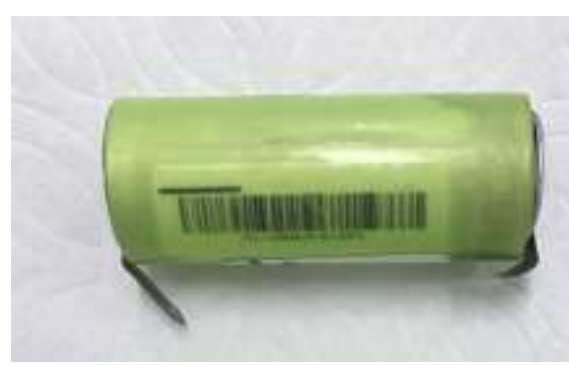

(a)

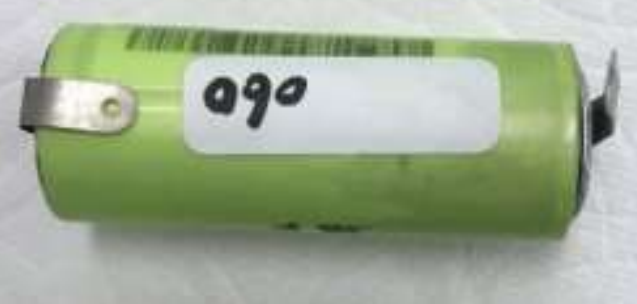

(b)

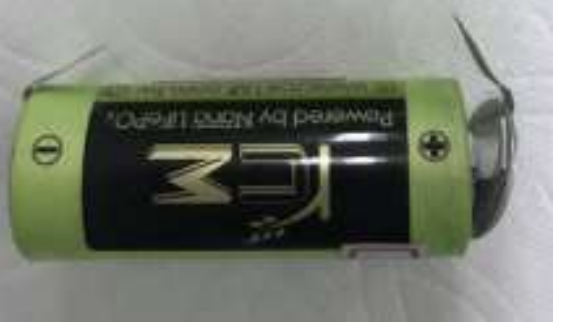

(c)

Fig. 5: Abnormal over-chargings (a) swelling on the end cover, (b) opening of the pressure relief valve, (c) Scorched surfaces on the battery packs with electrolyte leakage.

\section{Effects of Charging Current and SOH on Over-charging}

Figure 6 shows the variations of the terminal voltages of a lithium iron phosphate battery related to the accumulated charges when charged with different currents. The terminal voltage on the battery is obviously affected by the charging current. At first, a higher charging current results in a higher terminal voltage. After the flat-voltage region, however, the accumulated charges are nearly the same at the turning points of the voltage curves. Since then and before the abnormal over-charging, the rising slopes are almost identical to each other, indicating that the battery can be fast charged with a higher current under the limitation of an acceptable excessive charge.

The effect of the state-of-health on the terminal voltage is illustrated by Figure 7. In which, a brand-new battery with an $\mathrm{SOH}$ of $99.54 \%$ and an aged battery with an $\mathrm{SOH}$ of $85.1 \%$ are both charged by a constant current of $1 \mathrm{C}$. It is found that the voltage curve of the aged battery rises up much faster than that of the new battery, meaning that the existing capacity and thus the acceptable charge has been degraded for the aged battery. 


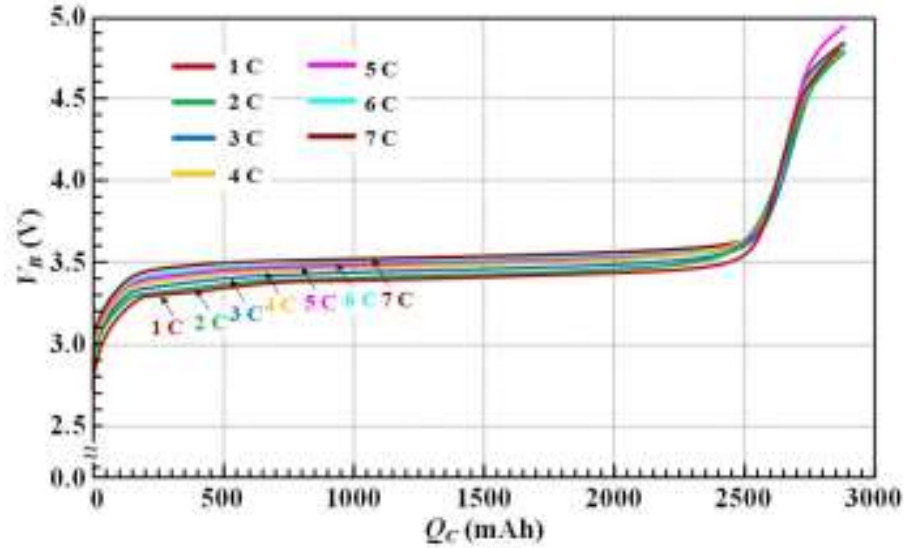

Fig. 6: Terminal voltages of a lithium iron phosphate battery related to the accumulated charges with different currents.

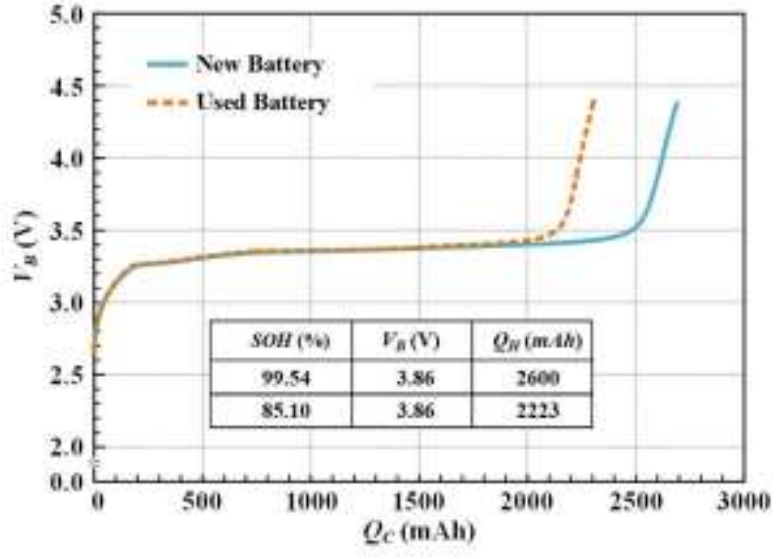

Fig. 7: Charging curves of terminal voltages with different SOHs.

\section{Over-charging with Excessive Charge and High Holding Voltage}

Figures 8 and 9 illustrate the experimental results for the over-charging with constant currents of $1 \mathrm{C}$ and $2 \mathrm{C}$, respectively. One can find that the terminal voltages of the tested batteries may rises up far above the gassing voltage. However, the batteries work well when the excessive amount charges are less than $11.5 \%$ of the existing capacity even though the terminal voltages are much higher than the gassing voltage. These two test results can evidence that the over charged batteries are more likely damaged by the excessive charge.

Alternatively, Figure 10 shows the experimental results from the tests with higher holding voltages at the $\mathrm{CV}$ stage. The tested batteries are all critically damaged by opening their pressure relief valves when they have suffered from a holding voltage higher than $4.5 \mathrm{~V}$ for a long period, which is the gassing voltage of the lithium iron phosphate battery. On the other hand, it is found that the pressure relief valves may open as the tested batteries are overly charged by an excessive charge of $300 \mathrm{mAh}$ even though the battery terminal voltage is below $4.5 \mathrm{~V}$.

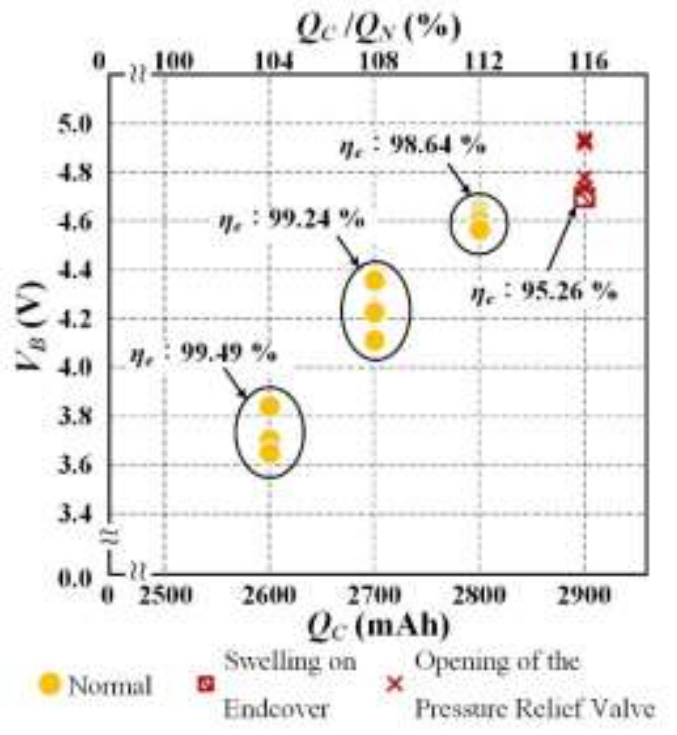

Fig. 8: Over-charging experiments by a constant current of $1 \mathrm{C}$.

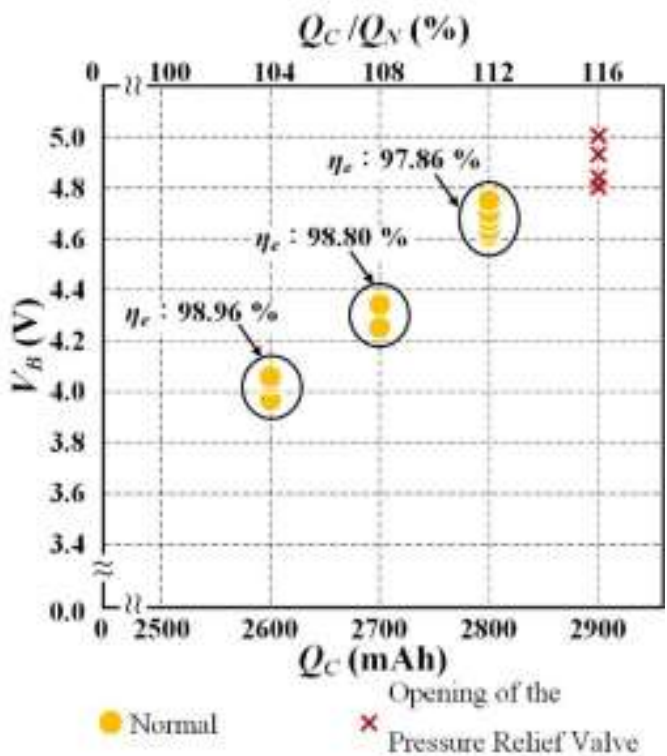

Fig. 9: Over-charging experiments by a constant current of $2 \mathrm{C}$. 


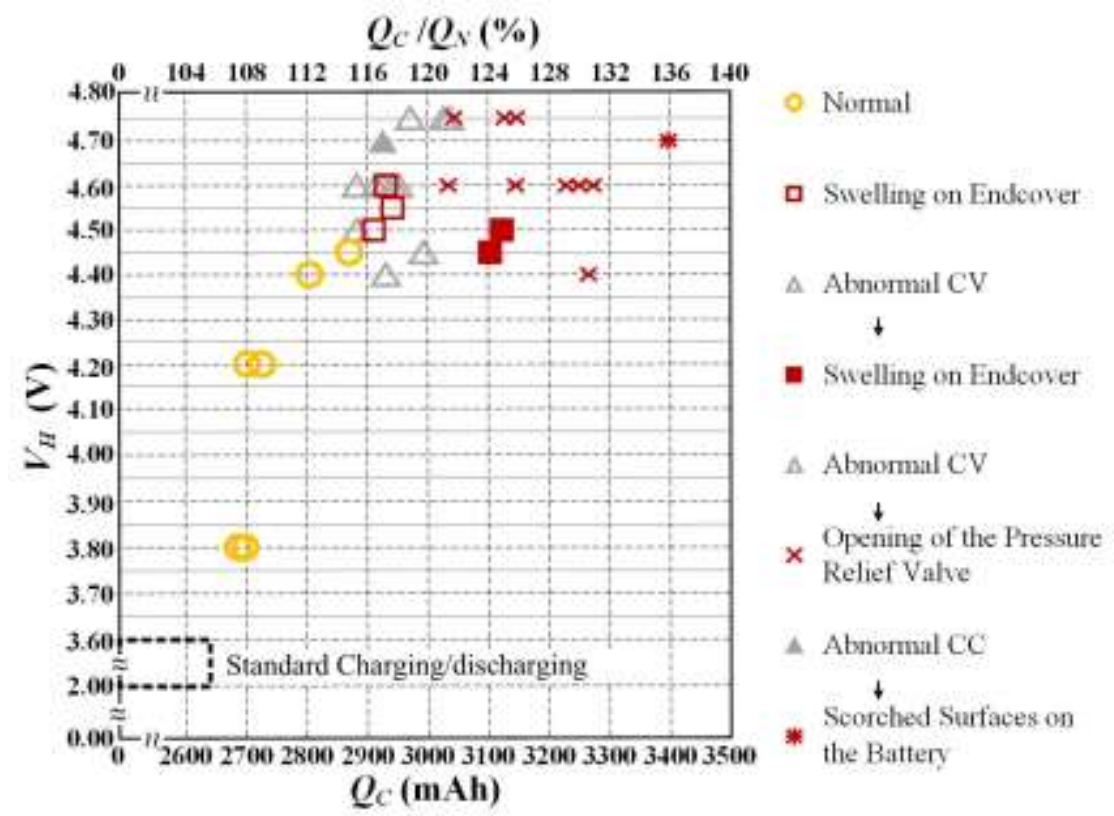

Fig. 10: Over-charging experiments with high holding voltages.

\section{SOH Tracking for Over-charged Batteries}

Before the over-charging experiments, the original capacities of a number of new lithium iron phosphate batteries are calculated by averaging the measured capacities obtaining from 5 full standard charging/discharging cycles. All of the tested batteries are completely discharged to be empty before being overly charged. The tested batteries are charged first by a constant current of $1 \mathrm{C}$ to higher holding voltages in a range from $3.7 \mathrm{~V}$ to $4.3 \mathrm{~V}$. As stated above, no damage is found on all tested batteries with the holding voltages in this range. After being overly charged, the cumulated charge volumes are higher than the original capacities with higher holding voltages. The higher the holding voltage is, the higher is the charged volume. By taking two hours for resting, the batteries are then completely discharged subsequently. As listed in Tables II and III, the releasable charges in the successive discharging phase are far less than the overly charged volumes but still higher the original capacity.

The SOHs of the overly charged batteries are tracked by a number of following standard charging/discharging cycles. As shown in Figures 11 and 12, the SOHs of the all tested batteries are found to be slightly higher than those of the batteries without over-charging after 50 full charging/discharging cycles. The SOHs of the overcharged batteries with the two most higher holding voltages of $4.3 \mathrm{~V}$ and $4.2 \mathrm{~V}$ are even higher than $100 \%$, indicating that there is no negative effect with these over-chargings.

Table 2: Charged and discharged volumes for first over-charging experiment.

\begin{tabular}{|c|c|c|c|c|}
\hline \multirow{2}{*}{$\begin{array}{c}\text { Holding } \\
\text { Voltage }\end{array}$} & \multicolumn{4}{|c|}{ Parameters } \\
\cline { 2 - 5 } & $Q_{H 1}$ & $Q_{A 1}$ & $Q_{R 1}$ & $Q_{H 1}^{\prime}$ \\
\hline $4.3 \mathrm{~V}$ & 2606 & 2902 & 2772 & 2686 \\
\hline $4.2 \mathrm{~V}$ & 2610 & 2829 & 2740 & 2669 \\
\hline $4.1 \mathrm{~V}$ & 2608 & 2732 & 2712 & 2651 \\
\hline $4.0 \mathrm{~V}$ & 2600 & 2708 & 2684 & 2633 \\
\hline $3.9 \mathrm{~V}$ & 2578 & 2694 & 2671 & 2629 \\
\hline $3.7 \mathrm{~V}$ & 2590 & 2667 & 2642 & 2616 \\
\hline
\end{tabular}

Table 3: Charged and discharged volumes for second over-charging experiment.

\begin{tabular}{|c|c|c|c|c|}
\hline \multirow{2}{*}{$\begin{array}{c}\text { Holding } \\
\text { Voltage }\end{array}$} & \multicolumn{4}{|c|}{ Parameters } \\
\cline { 2 - 5 } & $Q_{H 2}$ & $Q_{A 2}$ & $Q_{R 2}$ & $Q_{H 2}^{\prime}$ \\
\hline $4.3 \mathrm{~V}$ & 2640 & 2866 & 2726 & 2646 \\
\hline $4.2 \mathrm{~V}$ & 2640 & 2836 & 2714 & 2646 \\
\hline $4.1 \mathrm{~V}$ & 2623 & 2746 & 2693 & 2635 \\
\hline $4.0 \mathrm{~V}$ & 2620 & 2711 & 2675 & 2628 \\
\hline $3.9 \mathrm{~V}$ & 2619 & 2685 & 2662 & 2624 \\
\hline $3.7 \mathrm{~V}$ & 2614 & 2671 & 2642 & 2617 \\
\hline
\end{tabular}




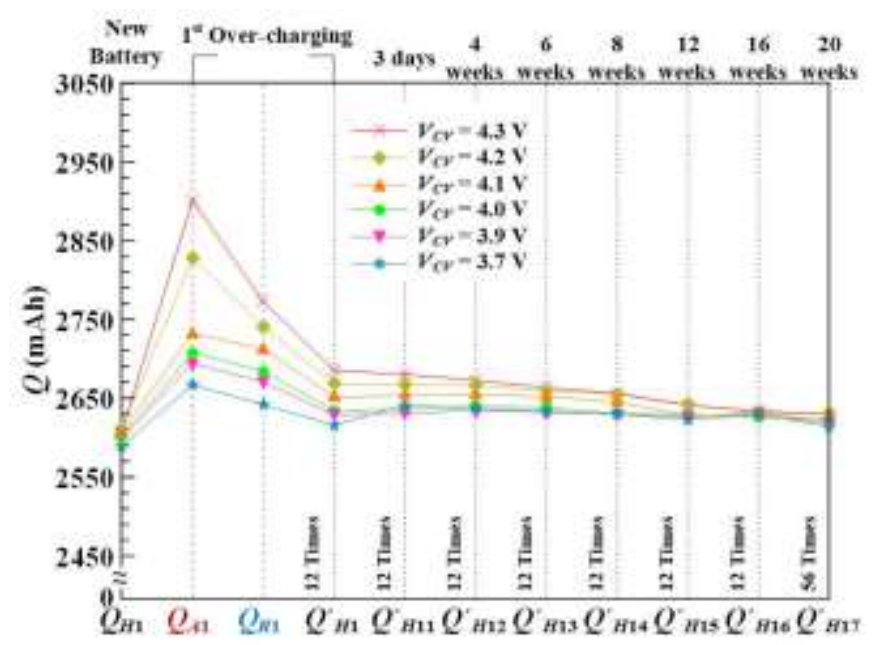

Fig. 11: SOH tracking after the first over-charging.

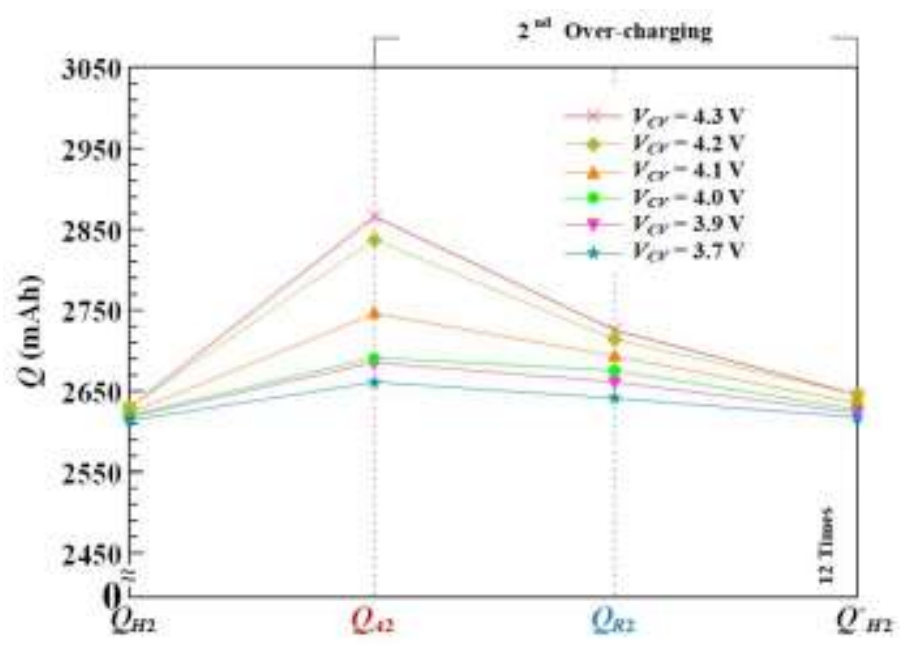

Fig. 12: SOH tracking after the second over-charging.

\section{Conclusion}

This research investigates the feasibility of extending usable capacity by overly charging the lithium iron phosphate battery with an acceptable amount of excessive charge. The over-charging experiments conducted on three brands of lithium iron phosphate batteries have demonstrated that serious damages with a swelling end, an opening pressure relief, and an electrolyte leakage may happen to the battery packs with either an extremely high holding voltage or an exceeding amount of surpassing charge. However, the harmful damage risks can be avoided by restricting the excessive capacity by $8 \%$ of the existing rated capacity and by limiting the holding voltage at the CV stage of the CC-CV charging method below $4.3 \mathrm{~V}$. The following $\mathrm{SOH}$ tracking reveals no significant negative effect on such over-charging experiments.

\section{Acknowledgements}

This work was supported by the Ministry of Science and Technology, Taiwan, under Grant 107-2221-E-110 -014 -and 107-2218-E-006 -003 -.

\section{References}

[1] S. Saxena, G. Sanchez, and M. Pecht, "Batteries in Portable Electronic Devices: A User's Perspective," in IEEE Industrial Electronics Magazine, vol. 11, no. 2, pp. 35-44, Jun. 2017.

[2] R. Xiong, J. Cao, Q. Yu, H. He, and F. Sun, "Critical Review on the Battery State of Charge Estimation Methods for Electric Vehicles," in IEEE Access, vol. 6, pp. 1832-1843, Dec. 2017.

[3] M. T. Lawder, B. Suthar, P. W. C. Northrop, S. De, C. M. Hoff, O. Leitermann, M. L. Crow, S. Santhanagopalan, and V. R. Subramanian, "Battery Energy Storage System (BESS) and Battery Management System (BMS) for Grid-Scale Applications," in Proceedings of the IEEE, vol. 102, no. 6, pp. 1014-1030, Jun. 2014.

[4] X. Zhang, H. Peng, H. Wang, and M. Ouyang, "Hybrid Lithium Iron Phosphate Battery and Lithium Titanate Battery Systems for Electric Buses," in IEEE Transactions on Vehicular Technology, vol. 67, no. 2, pp. 956-965, Feb. 2018.

[5] T. Horiba, "Lithium-Ion Battery Systems," in Proceedings of the IEEE, vol. 102, no. 6, pp. 939-950, Jun. 2014.

[6] D. Anseán, M. González, V. M. García, J. C. Viera, J. C. Antón, and C. Blanco, "Evaluation of $\mathrm{LiFePO}_{4} \mathrm{Batteries} \mathrm{for}$ Electric Vehicle Applications," in IEEE Transactions on Industry Applications, vol. 51, no. 2, pp. 1855-1863, Mar.Apr. 2015.

[7] R. C. Cope and Y. Podrazhansky, "The Art of Battery Charging," Fourteenth Annual Battery Conference on Applications and Advances. Proceedings of the Conference, Long Beach, CA, USA, 1999, pp. 233-235, Jan. 1999.

[8] S. S. Zhang, "The Effect of the Charging Protocol on the Cycle Life of a Li-ion Battery," in Journal of Power Sources, vol. 161, no. 2, pp. 1385-1391, Jul. 2006. 